Canad. Math. Bull. Vol. 20 (1), 1977

\title{
A COMMUTATIVITY THEOREM FOR NEAR-RINGS
}

BY

\author{
HOWARD E. BELL
}

A ring or near-ring $R$ is called periodic if for each $x \in R$, there exist distinct positive integers $n, m$ for which $x^{n}=x^{m}$. A well-known theorem of Herstein states that a periodic ring is commutative if its nilpotent elements are central [5], and Ligh [6] has asked whether a similar result holds for distributivelygenerated $(d-g)$ near-rings. It is the purpose of this note to provide an affirmative answer.

Our definition of near-ring assumes left distributivity, and the words "center" and "central" refer to multiplication. The term $R-R$ subgroup denotes an additive subgroup $S$ of $R$ such that $R S \subseteq S$ and $S R \subseteq S$. An element of a subnear-ring $T$ is called $T$-distributive if it is distributive with respect to addition in $T$. The near-ring $R$ is said to be the supplementary sum of its subnear-rings $A$ and $B$-denoted by $R=A+B$-if each element of $R$ can be uniquely represented in the form $a+b$, where $a \in A$ and $b \in B$.

TheOREM. Let $R$ be a distributively-generated near-ring with its nilpotent elements lying in the center. Then the set $N$ of nilpotent elements forms an ideal; and if $R / N$ is periodic, $R$ must be commutative.

\section{Proof of Theorem.}

LemMa 1. If $R$ is $a d-g$ near-ring in which nilpotent elements are central, then the set $N$ of nilpotent elements is an ideal.

Proof. Let $u_{1}, u_{2} \in N$ and $r \in R$. It is obvious that $r u_{1}$ and $u_{1} r \in N$; and the usual argument for commutative rings, which does not require additive commutativity, shows that $u_{1}-u_{2} \in N$. It remains to show that $N^{+}$is a normal subgroup of $R^{+}$, and this we do by induction on the degree of nilpotence.

If $u^{2}=0$ and $r \in R$, then $(r+u-r)^{2}=(r+u-r) r+(r+u-r) u-(r+u-r) r=$ $(r+u-r) r+u(r+u-r)-(r+u-r) r=0$. Now suppose $r+u-r$ is nilpotent for arbitrary $r \in R$ and $u \in N$ with index of nilpotence less than $k, k \geq 3$; and let $u \in N$ satisfy $u^{k}=0$. Then, letting $a=(r+u-r) r$ and proceeding as above, we have $(r+u-r)^{2}=a+\left(u r+u^{2}-u r\right)-a=(a+u r)+u^{2}-(a+u r)$, which is nilpotent since $\left(u^{2}\right)^{k-1}=0$. Thus $r+u-r \in N$.

Received by the editors April 4, 1975. 
Lemma 2. Let $R$ be a $d$-g near-ring with central nilpotent elements, and suppose that $R / N$ is periodic. Then

(1) for each $x \in R$, there exists an integer $n(x)>1$ for which $x-x^{n(x)}$ belongs to $N$;

(2) the commutator subgroup $R^{\prime}$ of $R^{+}$is contained in $N$;

(3) if $a, b \in R$ and $a b=0$, then $b a=0$.

Proof. The $d$-g near-ring $\bar{R}=R / N$ is periodic with no non-zero nilpotent elements; and it is easily shown that if $w \in \bar{R}$ and $w^{n}=w^{m}$ for $n>m>1$, then $w=w^{n-m+1}$. Therefore, for each $x \in R$, there exists an integer $n(x)>1$ for which $x-x^{n(x)} \in N \subseteq Z$-i.e. (1) holds. Since $\bar{R}$ is distributively-generated, Theorem 2 of [1] guarantees that $\bar{R}$ is additively commutative; and (2) follows at once. The proof of (3) is that of part $(A)$ of Lemma 3 in [2].

Part (3) of Lemma 2 guarantees that there is no distinction between left and right annihilators. Henceforth we shall denote the annihilator of an element $x$ of $R$ by $A(x)$.

Lemma 3. For any $R$ satisfying the hypotheses of Lemma 2, each of the following statements holds:

(1) If $e$ is an idempotent which is $S$-distributive for some $R-R$ subgroup $S$, then $e$ is central in $R$.

(2) For each non-zero central idempotent $e$ of $R, R^{\prime} \subseteq A(e)$.

(3) If $e_{1}, e_{2}, \ldots, e_{k}$ are pairwise-orthogonal central idempotents, then $e_{1} R+$ $\cdots+e_{k} R$ is a commutative ring.

(4) For each central idempotent e of $R, R=e R+A(e)$.

(5) If $R=A+B$ for a pair of orthogonal subnear-rings $A$ and $B$, and if the distributive element $d$ of $R$ is expressed as $d_{1}+d_{2}$ with $d_{1} \in A$ and $d_{2} \in B$, then $d_{2}$ is $B$-distributive.

Proof. (1) Let $e$ be an $S$-distributive idempotent of the $R-R$ subgroup $S$, and let $x$ be an arbitrary element of $R$. Since both ex and exe belong to $S$, we have $(e x-e x e) e=(e x) e-(e x e) e=0$; and by (3) of Lemma $2, e(e x-e x e)=$ ex - exe $=0$. Similarly, $x e-e x e=0$; thus $e \in Z$.

(2) Since $e$ is a central idempotent, $e R$ is a distributively-generated nearring with identity $e$ and has the property that $x-x^{n(x)}$ is central for each $x \in e R$. Thus, $e R$ is a commutative ring by Theorem 2 of [2]; and $e(x+y-x-$ $y)=0$ for all $x, y \in R$.

(3) Note that $e_{1} R+\cdots+e_{k} R+R^{\prime}$ is an $R-R$ subgroup containing the idempotent $e=\sum e_{i}$. Let $x=e_{1} r_{1}+\cdots+e_{k} r_{k}+r^{\prime}$, where $r_{1}, \ldots r_{k} \in R$ and $r^{\prime} \in R^{\prime}$; and use the distributivity and pairwise-orthogonality of the $e_{i}$ plus the fact that $R^{\prime}$ annihilates each $e_{i}$, to get the result that $e x=x e=\sum_{i=1}^{k} e_{i} r_{i}$. Thus, by (1) of Lemma $3, e$ is a central idempotent of $R$; and another appeal to Theorem 2 of [2] gives the result that $e R$ is a commutative ring. Since $e_{i} R \subseteq e R$ for each $i$, we are finished. 
(4) It is trivial to show that $r=e r+(-e r+r)$ is the unique representation of $r \in R$ in the form $a+b, a \in e R, b \in A(e)$.

(5) Let the distributive element $d$ be written in the form $d_{1}+d_{2}, d_{1} \in A$, $d_{2} \in B$. If $x, y$ are arbitrary elements of $B$, then $(x+y) d=(x+y) d_{1}+(x+y) d_{2}=$ $(x+y) d_{2}$ since $A$ and $B$ are orthogonal; on the other hand, by the distributivity of $d$ we get $(x+y) d=x d+y d=x d_{1}+x d_{2}+y d_{1}+y d_{2}=x d_{2}+y d_{2}$. Thus $d_{2}$ is distributive in $B$.

Lemma 4. Let $R$ satisfy the hypotheses of Lemma 2, and let $d$ be any distributive element of $R$. Then there exist an integer $n>1$ and a central idempotent $f$ of $R$ for which $d-d^{n} \in N$ and $d^{n} R=f R$.

Proof. By (1) of Lemma 2, there exists a positive integer $j$, which we may choose to be at least 3 , for which $d-d^{j} \in N$ and hence $d^{k}-d^{k+s(j-1)} \in N \subseteq Z$ for all positive integers $k$ and $s$. Since $d^{k}-d^{k+s(j-1)}$ commutes with $d+d$, we have $-d^{k+1+s(j-1)}+d^{k+1}=d^{k+1}-d^{k+1+s(j-1)}$, so that $d^{k+1}$ and $d^{k+1+s(j-1)}$ commute additively; and choosing $k+1=t(j-1), t=1,2, \ldots$, shows that all powers of $d^{i-1}$ are additively commutative. Thus the additive subgroup $S$ generated by the powers of $d^{j-1}$ is a $d-g$ near-ring with commutative addition, and hence a ring by a well-known theorem of Fröhlich [4]. Since the ring $S$ has the property that $x-x^{n(x)}$ is nilpotent for each $x \in S$, it is periodic by a theorem of Chacron [3]; therefore, there exist integers $p, q$ with $p>q$, such that $d^{p}=d^{q}$ and hence $d^{k}=d^{k+s(p-q)}$ for all non-negative integers $s$ and all $k \geq q$. In view of the last observation, we may assume that $p-q+1 \geq q>1$.

Let $n=p-q+1$. Then $d-d^{n} \in N$ by the proof of part (1) of Lemma 2 . Moreover, since $n \geq q$ and $n^{2}-n$ is divisible by $p-q$, we see that $\left(d^{n}\right)^{n}=d^{n}$. It follows at once that $f=d^{n(n-1)}$ is a distributive (hence central) idempotent and that $d^{n} R=f R$.

Proof of the Theorem. Let $x$ and $y$ be an arbitrary pair of elements of $\boldsymbol{R}$ and $d_{1}, d_{2}, \ldots, d_{t}$ distributive elements of $R$ which generate an additive subgroup containing both $x$ and $y$; suppose $d_{1}, \ldots, d_{k}$ are non-nilpotent and $d_{k+1}, \ldots, d_{t}$ are nilpotent. For each $i=1, \ldots, k$, choose an integer $n_{i}>1$ and a central idempotent $f_{i}$ such that $d_{i}-d_{i}^{n} \in N$ and $f_{i} R=d_{i}^{n_{i}} R$. In view of the fact that $R^{\prime} \subseteq N, \sum f_{i} R+N$ is a subnear-ring which contains both $x$ and $y$.

The next step is to construct a set of pairwise-orthogonal central idempotents $e_{1}, \ldots, e_{k}$ such that $\sum f_{i} R+N \subseteq \sum e_{i} R+N$. The case $k=1$ being immediate, we suppose that we have already obtained pairwise-orthogonal idempotents $e_{i}, \ldots, e_{s}$ (some of which may be trivial) such that $\sum_{i=1}^{s} f_{i} R+N \subseteq$ $\sum_{i=1}^{s} e_{i} R+N$. By repeated application of (4) of Lemma 3, write $R=A+B$, where $A=\sum_{i=1}^{s} e_{i} R$ and $B=\bigcap_{i=1}^{s} A\left(e_{i}\right)$; and let $f_{s+1}=g+h$, where $g \in A$ and $h \in B$. Now $(g+h)^{2}=(g+h) g+(g+h) h=g(g+h)+h(g+h)=g^{2}+h^{2}=g+h$, and by uniqueness of representation $h^{2}=h$. Since $h$ is $B$-distributive by (5) of 
Lemma $3, h$ must be central in $R$; and the fact that $R$ is distributivelygenerated and that $R^{\prime} \subseteq N$ shows that $f_{s+1} R=(g+h) R \subseteq g R+h R+N$. Denoting $h$ by $e_{s+1}$, and appealing to the inductive hypothesis, we get $\sum_{i=1}^{s+1} f_{i} R+N \subseteq$ $\sum_{i=1}^{s+1} e_{i} R+N$; and our construction is finished.

It remains only to show that $\sum_{i=1}^{k} e_{i} R+N$ is commutative. Accordingly let $u=e_{1} r_{1}+\cdots+e_{k} r_{k}+w$ and $v=e_{1} s_{1}+\cdots+e_{k} s_{k}+z$, where $w, z \in N$. Then, in view of the additive commutativity asserted by (3) of Lemma 3 ,

$$
\begin{aligned}
u v & =\sum_{i=1}^{k}\left(e_{1} r_{1}+\cdots+e_{k} r_{k}+w\right) e_{i} s_{i}+\left(\sum_{i=1}^{k} e_{i} r_{i}+w\right) z \\
& =\sum_{i=1}^{k} e_{i} r_{i} e_{i} s_{i}+\sum_{i=1}^{k} e_{i} w e_{i} s_{i}+\sum_{i=1}^{k} e_{i} r_{i} e_{i} z+w z ; \text { and } \\
v u & =\sum_{i=1}^{k} e_{i} s_{i} e_{i} r_{i}+\sum_{i=1}^{k} e_{i} z e_{i} r_{i}+\sum_{i=1}^{k} e_{i} s_{i} e_{i} w+z w .
\end{aligned}
$$

Now using the multiplicative commutativity of $\sum e_{i} R$ and the centrality of nilpotent elements, we see that $u v=v u$. This completes the proof of the theorem.

REMARK. If $R$ has 1 , it must in fact be a ring-this follows from Therorem 2 of [2]; however, in the absence of a multiplicative identity element, $R^{+}$need not be abelian.

\section{REFERENCES}

1. H. E. Bell, Near-rings in which each element is a power of itself, Bull. Australian Math. Soc. 2 (1970), 363-368.

2. - Certain near-rings are rings, J. London Math. Soc. (2) 4 (1971), 264-270.

3. M. Chacron, On a theorem of Herstein, Canadian J. Math. 21 (1969), 1348-1353.

4. A. Fröhlich, Distributively-generated near-rings, I, Ideal theory, Proc. London Math. Soc. (3) 8, (1958), 76-94.

5. I. N. Herstein, A note on rings with central nilpotent elements, Proc. Amer. Math. Soc. 5 (1954), 620.

6. S. Ligh, Some commutativity theorems for near-rings, Kyungpook Math. J. 13 (1973), 165-170.

Department OF MATHEMATICS

BROCK UNIVERSITY,

St. Catharines, Ontario 